\title{
Refining MDR-TB treatment regimens for ultra short therapy (TB-TRUST): study protocol for a randomized controlled trial
}

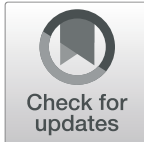

Taoping Weng ${ }^{1 \dagger}$, Feng Sun ${ }^{1 \dagger}$, Yang $\mathrm{Li}^{1 \dagger}$, Jiazhen Chen ${ }^{1}$, Xinchang Chen ${ }^{1}$, Rong Li ${ }^{1}$, Shijia Ge${ }^{1}$, Yanlin Zhao ${ }^{2^{*}}$ and Wenhong Zhang ${ }^{1 *}$

\begin{abstract}
Background: Multidrug-resistant tuberculosis (MDR-TB) are unsatisfied to treat, pressing more effective and innovative treatment regimens. New efficient regimens for MDR-TB have obtained high treatment success rates. However, those regimens without drug susceptibility testing (DST) are also likely to contribute to the emergence of resistance. Precision treatments guided by DST might optimize the patients' treatment outcome individually and minimize resistance amplification.

Methods: TB-TRUST is a phase III, multicenter, open-label, randomized controlled clinical trial of non-inferiority comparing the treatment success rate between the World Health Organization (WHO) shorter regimen and the refined ultra-short regimen for fluoroquinolones and second-line injectable drugs susceptible rifampicin-resistant TB. The control arm uses the WHO injectable-containing shorter regimen for 36-44 weeks depending on time of sputum smear conversion. The investigational arm uses a refined ultra-short regimen guided by molecular DST to pyrazinamide via whole-genome sequencing (WGS) to optimize the treatment of pyrazinamide-susceptible patients with levofloxacin, linezolid, cycloserine and pyrazinamide for 24-32 weeks and pyrazinamide-resistant with levofloxacin, linezolid, cycloserine and clofazimine for $36-44$ weeks. The primary outcome is the treatment success rate without relapse at 84 weeks after treatment initiation. Secondary outcomes include the time of sputum culture conversion and occurrence of adverse events. Assuming $a=0.025$ level of significance (one-sided test), a power of $80 \%$, a $<10 \%$ difference in treatment success rate between control arm and investigational (80\% vs. $82 \%$ ), and a $5 \%$ lost follow-up rate, the number of participants per arm to show non-inferiority was calculated as 177(354 in total).

\footnotetext{
* Correspondence: zhaoyanlin@chinatb.org; zhangwenhong@fudan.edu.cn; wenhongzhang_hs@126.com

†Taoping Weng, Feng Sun and Yang Li contributed equally to this work.

${ }^{2}$ National Center for Tuberculosis Control and Prevention, Chinese Center for

Disease Control and Prevention, Beijing, China

${ }^{1}$ Departments of Infectious Diseases, Huashan Hospital, Fudan University,

Shanghai 200040, China
}

(c) The Author(s). 2021 Open Access This article is licensed under a Creative Commons Attribution 4.0 International License, which permits use, sharing, adaptation, distribution and reproduction in any medium or format, as long as you give appropriate credit to the original author(s) and the source, provide a link to the Creative Commons licence, and indicate if changes were made. The images or other third party material in this article are included in the article's Creative Commons licence, unless indicated otherwise in a credit line to the material. If material is not included in the article's Creative Commons licence and your intended use is not permitted by statutory regulation or exceeds the permitted use, you will need to obtain permission directly from the copyright holder. To view a copy of this licence, visit http://creativecommons.org/licenses/by/4.0/ The Creative Commons Public Domain Dedication waiver (http://creativecommons.org/publicdomain/zero/1.0/) applies to the data made available in this article, unless otherwise stated in a credit line to the data. 
(Continued from previous page)

Discussion: Rapid molecular testing distinguishes patients who are eligible for shorter regimen with fluoroquinolone and the WGS-guided results shorten the treatment to 6 months for pyrazinamide susceptible patients. It's foreseeable that not only novel developed medicines, but also traditional powerful medicines with the susceptibility confirmed by DST are the key factors to ensure the effect of anti-MDR-TB drugs. As a DST-guided precision treatment, TB-TRUST are expected to optimize therapy outcome in more patients who cannot afford the expensive new medicines and minimize and even avoid resistance amplification with the rational use of anti-TB drugs.

Trail registration: ClinicalTrial.gov, NCT03867136. Registered on March 7, 2019.

Keywords: Tuberculosis, Multidrug-resistant tuberculosis, Multicenter, Randomized trial, Non-inferiority, Precision treatments, Ultra-short regimen

\section{Background}

As an ancient infectious disease, tuberculosis (TB) has gained new worldwide attention due to the emergence of drug resistance. About $20 \%$ of the TB strains have developed resistance to at least one anti-TB drug. In 2019, there were approximately 465,000 incident cases of rifampicin-resistant TB (RR-TB), of which $78 \%$ had multidrug-resistant TB (MDR-TB) [1].

MDR-TB is heavily challenging to treat, pressing more powerful and novel treatment regimens. Treatment for MDR-TB is largely carried out through standardized, empirical combination regimens. However, precision treatment guided by detailed DST results in improved individual outcomes [2]. Standardized regimens are expected to improve access to treatment, but they are also likely to contribute to the emergence of resistance. On the contrary, a DST-guided precision medicine will improve therapy outcome and minimize resistance amplification at the same time.

\section{Study of short regimen for MDR-TB treatment}

Treatment of MDR-TB remains challenging, with lengthy treatment durations and complex drug regimens. In 2010, Van et al. reported he Bangladesh regimen of 9month consisting of seven drugs for MDR-TB treatment, resulting in a treatment success rate of $87.9 \%$, marking the success of the short-term treatment for MDR-TB [3]. Subsequently, similar confirmatory studies were carried out in Cameroon [4] and Niger [5], all of which obtained extremely high treatment success rates over $89 \%$. In 2019, A.J. Nunn et al. published the first randomized controlled trial (RCT) study on short-term treatment of MDR-TB. The standardized shorter regimen of 9-11 months composed of 7 drugs with a treatment success rate of $78.8 \%$ was proved to be non-inferiority with the long-term program recommended by the World Health Organization (WHO) in 2011 [6]. After reviewing the results of the STREAM study and other observational studies, WHO released updated guidelines for MDR-TB in 2018 that introduced shorter regimen as an option for patients who have not been previously treated for more than 1 month with second-line medicines or have no evidence of resistance to fluoroquinolones and secondline injectable drugs (SLIDs) [7]. In 2020, Conradie F et al. published the outcome of Nix TB study [8]. They investigated the regimen of three oral drugs including bedaquiline, pretomanid and linezolid given for 26 weeks in patients with extensively drug-resistant (XDR) TB. Totally, 109 patients were enrolled in the study and at the end of treatment, 98 patients (90\%) had a favorable outcome, which suggested the combination of bedaquiline, pretomanid, and linezolid leading to a favorable outcome among a high percentage of patients with fluoroquinolones resistance.

In our early study, we showed that introducing molecular DST to pyrazinamide successfully improved the treatment outcome with the relapse-free success rate of $82.4 \%$ for pyrazinamide-susceptible cases and shortened the regimen to 12 months without any additional agents [9]. However, concerning its limits of nonrandomized design and relatively small sample sizes, we here launched this randomized controlled trial, Refining MDR-TB Treatment Regimens for Ultra Short Therapy (TB-TRUST), with the objective to evaluate the effectiveness and feasibility of a novel all-oral ultra-short regimen with the guidance of molecular DST to pyrazinamide.

\section{Innovative DST technology for fluoroquinolones, SLIDs and pyrazinamide}

Rapid testing of resistance to fluoroquinolones and SLIDs is of great significance for recognizing MDR-TB patients who are eligible and recommended in shorter regimens. Xpert ${ }^{\oplus}$ MTB/XDR (Cepheid, Sunnyvale, CA, USA) leverages the new 10-colour homogeneous detection within the GeneXpert system, which enables the detection of multiple mutations across several genes with detecting resistance to isoniazid, ethionamide, fluoroquinolones, amikacin, kanamycin and capreomycin. Xpert MTB/XDR testing can quickly select fluoroquinolones and SLIDs susceptible patients taking the most appropriate and precise shorter regimen [10]. 
Pyrazinamide resistance is associated with full-length mutations in pncA [11, 12], which can mainly detected through culturing strains of Mycobacterium tuberculosis (MTB). Whole-genome sequencing (WGS) is a viable and financially feasible tool for timely and comprehensive diagnosis of drug resistance, and our early study has proved it a promising approach to predict resistance to pyrazinamide with satisfactory accuracy, sensitivity and specificity of over $85.0 \%$ [13]. Our central laboratory, which has been studying resistant mechanism of MTB for a long time and has discovered many resistancerelated mutant sites [14-17], is able to interpret the result of the WGS to detect pyrazinamide resistance and review fluoroquinolones and SLIDs resistance tested by Xpert ${ }^{\circ}$ MTB/XDR, which makes precising and optimizing MDR-TB treatment regimen possible.

\section{Advanced RCT study with DST for pyrazinamide}

TB-TRUST is designed based on the trend of shorter and full oral treatment programs under DST guidance. Based on the molecular susceptibility testing platforms of Xpert ${ }^{\circ} \mathrm{MTB} / \mathrm{XDR}$ and WGS, a randomized controlled study is carried out. This study innovatively detects fluoroquinolone and SLIDs resistance to select opportune rifampicin-resistant/multidrug-resistant tuberculosis (RR/MDR-TB) patients and optimizes the regimen according to result of pyrazinamide resistance, comparing with the WHO shorter regimen as a standardized and non-DST guided control arm. This protocol describes the background, design and rationale of the TBTRUST trial.

\section{Methods}

\section{Study regimen and comparator}

This is a phase III, multicenter, open-label, randomized controlled clinical trial of non-inferiority with two arms, recruiting RR/MDR-TB patients susceptible to fluoroquinolones and SLIDs detected by $\mathrm{Xpert}^{\circ} \mathrm{MTB} / \mathrm{XDR}$. Recruited participants will be randomly divided into the ultra-short regimen as investigational arm and the WHO shorter regimen as control with a ratio of 1:1. Figure 1 shows the flow chart of TB-TRUST trail.

The ultra-short regimen consists of two periods. During the first 4-8 weeks, patients are waiting for the result of pyrazinamide DST and receive five agents including levofloxacin, linezolid, cycloserine, pyrazinamide and clofazimine. The result of molecular DST to pyrazinamide will be determined by two technical staff in the central laboratory of Huashan Hospital, Fudan University according to the established criteria that were reported previously in elsewhere [18, 19], refining the ultra-short regimen. After WGS results including pyrazinamide resistance are obtained, patients will be divided into two subgroups: pyrazinamide-susceptible patients and pyrazinamide-resistant patients. For pyrazinamidesusceptible patients, clofazimine will be discontinued while levofloxacin, linezolid, cycloserine and pyrazinamide are given until 24th week (prolonged to 28 or 32 weeks if smear has not converted by 16 or 20 weeks). The regimen for pyrazinamide-resistant patients consists of levofloxacin, linezolid, cycloserine and clofazimine given until 36th week (prolonged to 40 or 44 weeks if smear has not converted by 16 or 20 weeks). If the sputum culture is always negative resulting in the unavailability of WGS, the treatment regimen of the five drugs will be continued to 36 weeks (prolonged to 40 or 44 weeks if smear has not converted by 16 or 20 weeks).

The control regimen is WHO injectable-containing shorter regimen with two phases of treatment for 36-44 weeks. The first is the intensive phase of 16 weeks (extended up a maximum of 20 or 24 weeks in case of lack of smear conversion by 16 or 20 weeks), containing moxifloxacin, amikacin, prothionamide, pyrazinamide, high-dose isoniazid, ethambutol and clofazimine. The followed one is a continuation phase for 20 weeks with the following agents: moxifloxacin, pyrazinamide, ethambutol and clofazimine. Table 1 shows the doses and usage of each drug in the WHO shorter regimen and the ultra-short regimen.

\section{Site selection}

This trail is under the guidance of the China National Tuberculosis Defense Association, led by Huashan Hospital, Fudan University, Zhejiang Province Center for Disease Control and Prevention and Chinese Center for Disease Control and Prevention. The recruiting-cooperative units are 15 hospitals in nine provinces in China, namely Henan Infectious Disease Hospital, Xinjiang Uygur Autonomous Region Chest Hospital, Shenzhen Third People's Hospital, Hangzhou Red Cross Hospital, Ningbo Huamei Hospital of Chinese Academy of Sciences (Ningbo Second Hospital), Enze Hospital of Enze Medical Center (Group) in Taizhou, Zhejiang, the Affiliated Hospital of Shaoxing College of Arts and Sciences (Shaoxing Municipal Hospital), Guiyang Public Health Treatment Center, Wenzhou Central Hospital, Jiangxi Chest Hospital, Hunan Chest Hospital, Huaihua First People's Hospital, Shanxi Tuberculosis Prevention and Treatment Institute, Xuzhou Infection Disease Hospital, Yunan Infectious Diseases Hospital. A specialized auditing team will make the quality control of the cooperative units half a year.

\section{Patient eligibility criteria}

In addition to participate in this clinical trial voluntarily, the main eligibility criterion is smear-positive pulmonary RR/MDR-TB susceptible to fluoroquinolones and SLIDs identified by the Xpert $^{\circ}$ MTB/XDR platform. Patients 


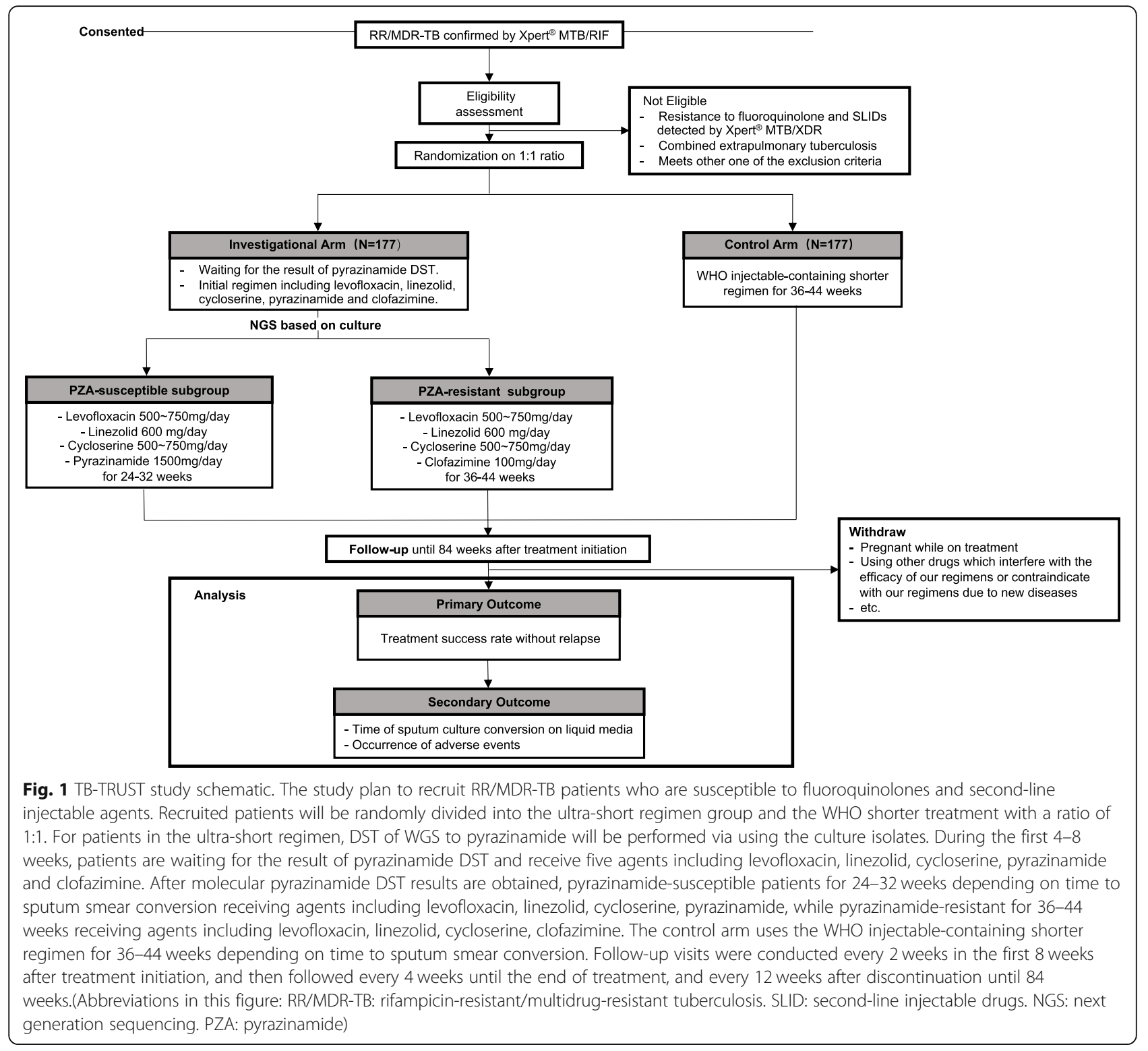

Table 1 TB-TRUST study regimen drugs and doses

\begin{tabular}{|c|c|c|c|}
\hline \multirow[t]{2}{*}{ Drug } & \multicolumn{2}{|l|}{ Weight group } & \multirow[t]{2}{*}{ Usage } \\
\hline & $\leq 50 \mathrm{~kg}$ & $>50 \mathrm{~kg}$ & \\
\hline Pyrazinamide & 1500 mg daily & & Once, before or after meal \\
\hline Levofloxacin & 500 mg daily & 750 mg daily & Once, before or after meal \\
\hline Linezolid & 600 mg daily & & Once, before or after meal \\
\hline Cycloserine & 500 mg daily & 750 mg daily & Once or divide in two, before or after meal \\
\hline Clofazimine & 100 mg daily & & Once, before or after meal \\
\hline Moxifloxacin & 400 mg daily & & Once, before or after meal \\
\hline Prothionamide & 500 mg daily & 750 mg daily & Once, before or after meal \\
\hline Ethambutol & 750 mg daily & 1000 mg daily & Once, before or after meal \\
\hline Amikacin & 600 mg daily & & Once, intramuscularly \\
\hline Isoniazid & 600 mg daily & & Once, before or after meal \\
\hline
\end{tabular}


will be excluded if they were in critical condition or with aspartate transaminase or alanine aminotransferase greater than five times the upper limit of normal, or blood creatinine greater than 1.5 times the upper limit of normal or HIV testing positive, considering the safety in the regimen. If patients are simultaneously applying the drugs that affect the efficacy of or contraindicated with this study drugs, they will be also excluded. Besides, pregnant or breastfeeding women are also excluded. Table 2 shows the detailed inclusion and exclusion criteria. Those patients are pregnant while on treatment and using other drugs which interfere with the efficacy of our regimens or contraindicate with our regimens due will withdraw, who will then be treated by Chinese national guideline for RR/MDR-TB.

\section{Recruitment process}

RR/MDR-TB patients identified by Xpert ${ }^{\circ}$ MTB/RIF assay (Cepheid, Sunnyvale, CA, USA) are screening to ascertain whether they satisfy the other eligibility criteria for enrolment. The follow-up content of this trial during the first screening including medical history collection, personal information collection, signed informed consent, physical examination, clinical evaluation, height and weight measuring, hearing examination, sputum smear, sputum culture, liver function, AIDS testing, blood sugar, blood potassium, electrocardiogram, chest radiography, urine routine, peripheral neuropathy brief examining with the use of a Brief Peripheral Neuropathy rating scale and ophthalmologic examination, including assessment of visual acuity and color vision. After screening procedures finished, potentially eligible patients are performed by Xpert ${ }^{\circ}$ $\mathrm{MTB} / \mathrm{XDR}$ to detect the resistance of fluoroquinolone and SLIDs, those without evidence of resistance to fluoroquinolone and SLIDs are recruited into this trial.

\section{Treatment allocation}

The Patients who meet the eligibility criteria will be registered online and managed by an authorized doctor in every branch centers. And then participants will be assigned to the study arm based on an 1:1 randomization ratio. The randomization is performed through the online central randomization system stratified by the study sites (sponsored by Clinflash, China), and the treatment plan is notified to the branch centers by the central laboratory of Huashan Hospital, Fudan University.

\section{Duration of follow-up}

After screening and baseline assessment, follow-up visits were conducted every 2 week in the first 8 weeks, and

Table 2 TB-TRUST inclusion and exclusion criteria

\begin{tabular}{|c|c|}
\hline & Detailed Description \\
\hline Inclusion criteria & $\begin{array}{l}\text { 1) Willing to participate in trial treatment, follow-up and sign informed consent } \\
\text { 2) Age 18-70 years old } \\
\text { 3) Smear-positive pulmonary tuberculosis with resistance to rifampicin confirmed by Xpert }{ }^{\circledR} \text { MTB/RIF } \\
\text { (Cepheid, Sunnyvale, CA, USA) } \\
\text { 4) Willing to carry out HIV testing } \\
\text { 5) If a non-menopausal woman, willing to use or have used effective contraception during treatment } \\
\text { 6) Have an identifiable address and stay in the area during the study period } \\
\text { 7) Willing to follow the follow-up study procedure after the follow-up. }\end{array}$ \\
\hline Exclusion criteria & $\begin{array}{l}\text { 1) Resistant to second-line injection confirmed by Xpert }{ }^{\circledR} \text { MTB/XDR (Cepheid, Sunnyvale, CA, USA) } \\
\text { 2) Resistant to fluoroquinolone confirmed by Xpert }{ }^{\oplus} \text { MTB/XDR } \\
\text { 3) Combined extrapulmonary tuberculosis } \\
\text { 4) HIV antibody positive and AIDS patients } \\
\text { 5) Severe patients and impossible to survive for more than } 16 \text { weeks according to the judgment of the } \\
\text { research physician } \\
\text { 6) Have been pregnant or breastfeeding } \\
\text { 7) Unable to attend or follow treatment or follow-up time } \\
\text { 8) Cannot take oral medications } \\
\text { 9) Patients with impaired liver function (hepatic encephalopathy, ascites; total bilirubin is more than } \\
\text { 2 times higher than the upper limit of normal; ALT or AST is more than } 5 \text { times the upper limit of normal) } \\
\text { 10) Blood creatinine is more than } 1.5 \text { times the upper limit of normal } \\
\text { 11) The investigator believes that there are any social or medical harms that expose to the participants } \\
\text { 12) Simultaneously apply the drugs (glucocorticoids, interferons) that affect the efficacy of this study; } \\
\text { and apply the following drugs contraindicated with the study regimens, including non-steroidal } \\
\text { anti-inflammatory drugs, monoamine oxidase inhibitors (phenethyl hydrazine, different Carbofurs et al), } \\
\text { direct or indirect sympathomimetic drugs (such as pseudoephedrine), vasopressor drugs (such as: adrenaline, } \\
\text { norepinephrine), dopamine drugs (such as: dopamine, dobutamine), 5-HI reuptake inhibitor, a tricyclic } \\
\text { antidepressant, a 5-HTI receptor antagonist (amitriptyline), meperidine or buspirone } \\
\text { 13) Being allergic or intolerant of any study drug } \\
\text { 14) Currently participating in another drug clinical trial } \\
\text { 15) QTc interval } \geq 500 \text { ms during screening } \\
\text { 16) Hemoglobin is less than } 90 \text { g/L or platelet is less than } 75^{*} 10 \wedge 9 / L \\
\text { 17) Have epilepsy, severe depression, irritability or psychosis } \\
\text { 18) Alcohol abuse }\end{array}$ \\
\hline
\end{tabular}


then every 4 weeks until the end of treatment, and every 12 weeks at the period of post-treatment until 84 weeks after treatment initiation. During the follow-up, physical examination, clinical assessment, weight measurement, sputum smear, sputum culture, blood and urine routine, liver function, renal function, electrocardiogram, brief peripheral neuropathy screen, ophthalmologic examination and some other tests will be evaluated. Detailed schedule of patient monitoring exams and laboratory tests will be shown in the additional file. To promote adherence to intervention, patients are asked to keep a drug-tablet taking diary and they will get allowance after completion of follow-up. Study enrollment began in June 2020 and is expected to finished in about half a years.

\section{Sample size assumptions}

This sample size was calculated using the PASS 11 system (NCSS, Version: 11.0.10). Assuming $\alpha=0.025$ level of significance (one-sided test), a power of $80 \%$ and a non-inferiority margin of $10 \%$ between the control and investigational arms (80\% versus $82 \%$ favorable outcome), the number of participants needed per arm to show non-inferiority of the investigational regimen was calculated as 168 . Considering the $5 \%$ lost follow-up rate, 177 patients were required for each are, so the total sample size of the two arms was considered to be 354 .

\section{Data collection and quality management}

Clinic staff in the cooperative hospitals or centers will use the specialized website and data collection forms for study management to record data. All data access in websites will be controlled by unique usernames and passwords and staff will have access restricted to the functionality and data that are appropriate for their role in the study. Central Coordinating Office (CCO) staff will be responsible for provision of the relevant website and case report forms. CCO will check the eligibility of patients and follow-up data integrity regularly. After patients complete the trial, $\mathrm{CCO}$ will lock the file for further generation of data extracts for analysis. Only three main investigators of Huashan Hospital, Fudan University can have access to the final trial dataset and report the results of the trial via publication.

\section{Adverse event (AE) management}

The grading system for AE has been established. To provide the advice to complicated event management, we build a highly experienced clinician team which includes a pulmonologist, an infectious diseases expert, a nephrologist, a psychiatrist, a cardiologist, a hepotologist, a hematologist, a rheumatologist, and an experienced nurse. Severe AE will be reported to the center within $24 \mathrm{~h}$ and discussed by the committee. $\mathrm{AE}$ and the adjustment or withdraw of drugs will be recorded and checked by CCO.

\section{External quality control of phenotypic DST}

Baseline M.tuberuclosis strains from each patients enrolled in this study were sent to the National Tuberculosis Reference Laboratory of Chinese Center for Disease Control and Prevention in accordance with international transportation guidelines. A total of twelve anti-tuberculosis drugs susceptibility, including ofloxacin, moxifloxacin, rifampin, amikacin, rifabutin, para-aminosalicylic acid, ethionamide, isoniazid, kanamycin, ethambutol, streptomycin, and cycloserine were measured with the use of the Sensitire MYCOTB MIC Plate (MYCOTB) on one 96-wells plate.

\section{Assessment and analysis of outcome}

The primary objective is to compare the treatment success rate without relapse between the WHO injectable-containing shorter regimen group and the refined ultra-short regimen group at 84 weeks after treatment initiation. Treatment outcomes will be classified into favorable outcome which means successful treatment and unfavorable outcome. Table 3 describes the definition of favorable and unfavorable outcome. Secondary outcomes including the time of sputum culture conversion and AEs are evaluated as well.

The results of this trial for efficacy outcomes will be analyzed based on both modified intention-to-treat $(m I T T)$ and per protocol $(P P)$ approaches with a primary consideration for $m I T T$ results. For the primary outcome analysis, the upper bound of the $95 \%$ confidence interval of the difference in proportion of favorable outcome between the WHO shorter regimen and the refined ultrashort regimen must be less than 10\% (the margin of non-inferiority) in both the $m I T T$ and the PP populations for the ultra-short regimen to be declared noninferior to the WHO shorter regimen group.

The analysis of sputum smear conversion will base on the conversion time after treatment initiation and the median time will be estimated in each group using the Kaplan-Meier method, and the difference in the distribution of time to culture conversion of the two regimens will be compared in a Cox proportional-hazard model. All AEs, serious AEs (SAEs) and treatment interruption due to adverse events will be collected and documented, and the proportion per type of AEs in the two regimens will be compared using the chi-square test or Fisher's exact test.

\section{Confidentiality}

Participants' personal information will be limited to only information necessary for outcome evaluation of study regimen with laws on privacy protection and guaranteeing of confidentiality. Paper documents containing 
Table 3 Definition of the favorable and unfavorable outcomes in TB-TRUST study

\begin{tabular}{ll}
\hline Outcome & Description \\
\hline Favorable outcome & A participant's outcome will be classified as favorable if their last two culture results are negative unless \\
they have previously been classified as unfavorable. These two cultures must be taken on separate visits \\
(on different days); the latest of which being within the Week 84 window (that is no more than 6 weeks \\
before 84 weeks since randomization but with no upper bound). \\
- Participants that don't have a culture result within the Week 84 window because they were unable to \\
produce sputum, will be classified as favorable if their last two cultures before the Week 84 window are \\
negative and they have not previously been classified as unfavorable; such participants will be identified \\
separately in tables. \\
A participant's outcome will be classified as unfavorable if: \\
- They are discontinued from their allocated study treatment and subsequently restarted on a different \\
MDR-TB regimen; \\
- Treatment is extended beyond the scheduled end of treatment for any reason other than making up \\
of days when no treatment was given (missed treatment) for a maximum of 8 weeks. A maximum of \\
14 days of extra treatment (irrespective of reason) is acceptable before it is classified as treatment extension. \\
In addition, if the phase of treatment has been extended for delayed sputum conversion (maximum \\
8-week extension permitted) the scheduled end of treatment will also be extended by the same amount; \\
- They are restarted on any MDR-TB treatment after the scheduled end of treatment, but before 84 weeks \\
after randomization; \\
- More than 1 drug has been changed for any reason; \\
- They die at any point during treatment or follow-up; \\
- At least one of their last two culture results, from specimens taken on separate occasions, is positive;
\end{tabular}

participants' data will be locked in a specialized office in cooperative hospitals. Digital documents will be stored in password-protected files on website. Access to study documents will be restricted to authorized personnel only.

\section{Discussion}

China accounted for $14 \%$ of the global total MDR/RR$\mathrm{TB}$ in 2019, while treatment of RR/MDR-TB success rate only reached to $57 \%$ globally [1]. To improve the treatment outcome of RR/MDR-TB and optimize the regimens are urgently needed to work together worldwide.

Exploration of initiatives for MDR-TB started decades ago, but only increased in size and scope in the past few years. In 2020, WHO consolidated guidelines on TB (Module 4) revised current policy recommendations on treatment strategy for drug-resistant TB (DR-TB) [20]. The 2020 recommendations has witnessed two important trends in the MDR-TB treatment. One is the shorter duration of 6-12 months, founded on the Bangladesh regimen and the STREAM study. Another is the all-oral regimen in the strength of the effective novel and repurposed agents, following the promising results of the NixTB study. As a new recommendation, the shorter regimen where the injectable agent is replaced by bedaquiline is highly preferred for the patients with resistance to fluoroquinolones ruled out. However, the revised all-oral bedaquiline-containing regimen still includes seven agents, most of which are Group $\mathrm{C}$ agents. One pitfall of the regimen is the underappreciated role of the drug susceptibility testing (DST) for other agents except fluoroquinolones.

At present, a number of studies of shorter regimen for MDR-TB including MDR-END [21], endTB [22] and so on are carried out. The trends of MDR-TB treatment are short-term and full oral. However, how to optimize the patient's treatment process and outcome based on DST has not been emphasized. Introducing rapid DST and more effective oral drugs to ensure drugs' effect is a potential way to shorten and optimize the treatment. Especially DST of pyrazinamide, which plays a key role in reducing $\mathrm{TB}$ relapse rates and shortening the course of the disease treatment from 9 to 12 months to 6 months due to its powerful sterilizing activity $[23,24]$, is valuable and vital in those published studies $[9,25]$.

Our early study [9] provides excellent treatment success rate of precise regimen based on DST. It was the first prospective study that supported the superiority of molecular DST in guiding treatment decisions and refining treatment regimens. But the study was not designed in randomization, which means opinion was divided as to the impact of potential biases and whether these results could be replicated in different settings remains considering. TB-TRUST study is set up as a high- level evidence RCT study to evaluate the treatment outcome of precision treatment non-inferior to WHO shorter regimen, which uses rapid molecular testing to identify patients who can take shorter regimen with fluoroquinolone and shortens the treatment to 6 months for pyrazinamide sensitive patients based on the WGS result without introducing any new anti-TB medicines. 
The 2018 WHO guidelines for DR-TB [7] proposed a three-drug combination treatment plan based on moxifloxacin, linezolid, and bedaquiline, which represented the arrival of the era of totally oral treatment. Nix-TB study with three oral drugs in 26 weeks achieved the favorable outcome in $90 \%$ patients, which means ensured effective drugs can achieve a 6-month shorter treatment regimen without too many drug, even for highly MDRTB. However, not only novel developed medicines, but also traditional effective medicines with the susceptibility confirmed by DST are the key factors to ensure the effect of anti-MDR-TB drugs. Furthermore, a DST-guided precision treatment with traditional agents optimizes therapy outcome in more patients who cannot afford the expensive new medicines. With the rational use of antituberculosis drugs, precision treatment can minimize and even avoid resistance amplification.

TB-TRUST study has few limitations. As a full-oral shorter regimen, without introducing bedaquiline is regrettably. Bedaquiline-containing regimens have achieved high conversion and success rates of MDRand XDR-TB trials in published studies [8, 26, 27] and it is a popular and important agent in recent shorter regimen trials [22]. However, most Chinese patients cannot afford bedaquiline due to the high price and different medical insurance reimbursement ratio, which has affected its promotion in China. TB-TRUST study hopes to prove that accessible medicines are also effective with the guidance of DST and we believe that a bedaquilinecontaining regimen under the precise DST will achieve better efficacy. Moreover, WGS based on TB cultured isolations is an accurate and effective method for DST of pyrazinamide at present. However, long period of acquiring the cultured isolations limits the acquisition of DST in time. To guide the design of regimens immediately and precisely in the beginning of therapy, it's expectant that WGS or other innovative technology can be performed directly on clinical specimens without cultured isolations [28].

\section{Supplementary Information}

The online version contains supplementary material available at https://doi. org/10.1186/s12879-021-05870-w.

\section{Additional file 1.}

\footnotetext{
Abbreviations

AEs: Adverse events; AST: Aspartate transaminase; ALT: Alanine aminotransferase; BPNS: Brief peripheral neuropathy rating scale; CCO: Central coordinating office; DR-TB: Drug-resistant tuberculosis; DST: Drug susceptibility testing; MDR-TB: Multidrug-resistant tuberculosis; m/TT: Modified intention-to-treat; MTB: Mycobacterium tuberculosis; PP: Per protocol; RCT: Randomized controlled Trial; RR-TB: Randomized controlled trial; SAEs: Serious adverse events; SLIDs: Second-line injectable drugs; WGS: Whole-genome sequencing; XDR: Extensively drug-resistant
}

\section{Acknowledgements}

We thank all physicians from 15 centers that participated in this study for patient enrollment and follow-up. It's sincere thankful for all cooperative pharmaceutical factories to donate all anti-MDR-TB medicines for every participant, including the National Thirteenth Five-Year Plan for Science and Technology and Chinese pharmaceutical companies including Zhejiang Hisun Pharmaceutical Co., Ltd., Beijing Fuyuan Pharmaceutical Co., Ltd., Shanxi Liye Pharmaceutical Co., Ltd., Chongqing Winbond Pharmaceutical Co., Ltd., Hangzhou Minsheng Pharmaceutical Co., Ltd., Shenyang Hongqi Pharmaceutical Co., Ltd., Daiichi Sankyo Pharmaceutical (Beijing) Co., Ltd. And we thank Cepheid (Sunnyvale, CA, USA) for providing the platform of Xpert ${ }^{\oplus}$ MTB/XDR. We also thank our statistician, Junyan Zhang (Clinical Epidemiology and Evidence-Based Medicine, Shanghai Medical Association) for assistance in sample size assumption and data analysis. And we would like to thank all our patients for participating in this study and contributing to global evidence.

\section{Authors' contributions}

All authors read and approved the final manuscript. TW,YL, FS and WZ contributed to the conception and design of the study. TW and FS contributed towards the development of the electronic medical record system for data collection. TW and YL drafted the manuscript and all authors contributed to subsequent revisions. JC, XC, RL and SG contributed to edit the manuscript. WZ and $\mathrm{YZ}$ contributed towards the critical revision of the article.

\section{Funding}

The TB-TRUST study was supported by a grant from National Major Science and Technology Projects of China (2018ZX10722301-003) and Clinical Research Plan of SHDC (No. SHDC2020CR1011B). The funder and donors had no role in study design, data collection and analysis, decision to publish, or preparation of the manuscript.

\section{Availability of data and materials}

This is a study protocol manuscript, Not applicable.

\section{Ethics approval and consent to participate}

The trial received ethical approval from the Ethics Advisory Group of Huashan Hospital, Fudan University (approval number KY2019-416). Prior to undergoing any study-specific procedures, participants must provide signed and dated written informed consent to participate this trail, agree to collection and use of their data.

Consent for publication

Not applicable.

\section{Competing interests}

The authors declare that they have no conflict of interests.

Received: 25 November 2020 Accepted: 4 February 2021

Published online: 17 February 2021

\section{References}

1. WHO. Global tuberculosis report 2020. Geneva: World Health Organization; 2020. https:/www.who.int/publications///item/9789240013131. Accessed 11 Nov 2020

2. Cox H, Hughes J, Black J, Nicol MP. Precision medicine for drug-resistant tuberculosis in high-burden countries: is individualised treatment desirable and feasible? Lancet Infect Dis. 2018;18(9):e282-7.

3. Van Deun A, Maug AKI, Salim MAH, Das PK, Sarker MR, Daru P, et al. Short, highly effective, and inexpensive standardized treatment of multidrugresistant tuberculosis. Am J Respir Crit Care Med. 2010;182(5):684-92.

4. Kuaban C, Noeske J, Rieder HL, Ait-Khaled N, Abena Foe JL, Trébucq A. High effectiveness of a 12-month regimen for MDR-TB patients in Cameroon. Int J Tuberc Lung Dis Off J Int Union Tuberc Lung Dis. 2015;19(5):517-24.

5. Piubello A, Harouna SH, Souleymane MB, Boukary I, Morou S, Daouda M, et al. High cure rate with standardised short-course multidrug-resistant tuberculosis treatment in Niger: no relapses. Int J Tuberc Lung Dis Off J Int Union Tuberc Lung Dis. 2014;18(10):1188-94. 
6. Nunn AJ, Phillips PPJ, Meredith SK, Chiang C-Y, Conradie F, Dalai D, et al. A trial of a shorter regimen for rifampin-resistant tuberculosis. N Engl J Med. 2019;380(13):1201-13.

7. Rapid Communication. Key changes to treatment of multidrug- and rifampicin-resistant tuberculosis (MDR/RR-TB). Geneva: World Health Organization; 2018. https://www.who.int/publications/i/item/WHO-CDS-TB-2 018.18. Accessed 11 Nov 2020

8. Conradie F, Diacon AH, Ngubane N, Howell P, Everitt D, Crook AM, et al. Treatment of Highly Drug-Resistant Pulmonary Tuberculosis. N Engl J Med. 2020;382(10):893-902.

9. Sun F, Li Y, Chen Y, Guan W, Jiang X, Wang X, et al. Introducing molecular testing of pyrazinamide susceptibility improves multidrug-resistant tuberculosis treatment outcomes: a prospective cohort study. Eur Respir J. 2019;53(3):1801770.

10. New Rapid Molecular Test For Tuberculosis Can Simultaneously Detect Resistance To First- And Second-Line Drugs - Jul 16, 2020. Available from: http://cepheid.mediaroom.com/2020-07-16-New-Rapid-Molecular-Test-ForTuberculosis-Can-Simultaneously-Detect-Resistance-To-First-And-SecondLine-Drugs. Accessed 11 Nov 2020.

11. Yadon AN, Maharaj K, Adamson JH, Lai Y-P, Sacchettini JC, loerger TR, et al. A comprehensive characterization of PncA polymorphisms that confer resistance to pyrazinamide. Nat Commun. 2017:8(1):588.

12. Liu W, Chen J, Shen Y, Jin J, Wu J, Sun F, et al. Phenotypic and genotypic characterization of pyrazinamide resistance among multidrug-resistant Mycobacterium tuberculosis clinical isolates in Hangzhou, China. Clin Microbiol Infect. 2018;24(9):1016.e1-5.

13. Chen X, He G, Wang S, Lin S, Chen J, Zhang W. Evaluation of wholegenome sequence method to diagnose resistance of 13 anti-tuberculosis drugs and characterize resistance genes in clinical multi-drug resistance mycobacterium tuberculosis isolates from China. Front Microbiol. 2019;10: 1741.

14. Shi W, Chen J, Feng J, Cui P, Zhang S, Weng X, et al. Aspartate decarboxylase (PanD) as a new target of pyrazinamide in Mycobacterium tuberculosis. Emerg Microbes Infect. 2014;3(1):1-8.

15. Zhang S, Chen J, Cui P, Shi W, Zhang W, Zhang Y. Identification of novel mutations associated with clofazimine resistance in Mycobacterium tuberculosis: table 1. J Antimicrob Chemother. 2015;70(9):2507-10.

16. Zhang S, Chen J, Shi W, Liu W, Zhang W, Zhang Y. Mutations in panD encoding aspartate decarboxylase are associated with pyrazinamide resistance in Mycobacterium tuberculosis. Emerg Microbes Infect. 2013;2(1):1-5.

17. Zhang S, Chen J, Cui P, Shi W, Shi X, Niu H, et al. Mycobacterium tuberculosis mutations associated with reduced susceptibility to linezolid. Antimicrob Agents Chemother. 2016;60(4):2542-4.

18. Zhang Y, Zhang J, Cui P, Zhang Y, Zhang W. Identification of novel efflux proteins Rv0191, Rv3756c, Rv3008, and Rv1667c involved in pyrazinamide resistance in mycobacterium tuberculosis. Antimicrob Agents Chemother. 2017:61(8):e00940-17 e00940-17.

19. Zhang S, Chen J, Shi W, Cui P, Zhang J, Cho S, et al. Mutation in ClpC1 encoding an ATP-dependent ATPase involved in protein degradation is associated with pyrazinamide resistance in Mycobacterium tuberculosis. Emerg Microbes Infect. 2017;6(1):1-2.

20. WHO. WHO Consolidated Guidelines on Tuberculosis, Module 4: Treatment - Drug-Resistant Tuberculosis Treatment. Geneva: World Health Organization; 2020. https://www.who.int/publications/i/item/978924000704 8. Accessed 11 Nov 2020

21. Lee M, Mok J, Kim DK, Shim TS, Koh W-J, Jeon D, et al. Delamanid, linezolid, levofloxacin, and pyrazinamide for the treatment of patients with fluoroquinolone-sensitive multidrug-resistant tuberculosis (treatment shortening of MDR-TB using existing and new drugs, MDR-END): study protocol for a phase II/III, multicenter, randomized, open-label clinical trial. Trials. 2019;20(1):57.

22. Khan U, Huerga $H$, Khan AJ, Mitnick CD, Hewison C, Varaine F, et al. The endTB observational study protocol: treatment of MDR-TB with bedaquiline or delamanid containing regimens. BMC Infect Dis. 2019;19(1):733.

23. Gopal P, Grüber G, Dartois V, Dick T. Pharmacological and molecular mechanisms behind the sterilizing activity of pyrazinamide. Trends Pharmacol Sci. 2019;40(12):930-40.

24. Li S-Y, Tasneen R, Tyagi S, Soni H, Converse PJ, Mdluli K, et al. Bactericidal and sterilizing activity of a novel regimen with Bedaquiline, Pretomanid, Moxifloxacin, and pyrazinamide in a murine model of tuberculosis. Antimicrob Agents Chemother. 2017;61(9):e00913-7 e00913-17.
25. Njire M, Tan Y, Mugweru J, Wang C, Guo J, Yew W, et al. Pyrazinamide resistance in mycobacterium tuberculosis: review and update. Adv Med Sci. 2016;61(1):63-71.

26. Pym AS, Diacon AH, Tang S-J, Conradie F, Danilovits M, Chuchottaworn C, et al. Bedaquiline in the treatment of multidrug- and extensively drugresistant tuberculosis. Eur Respir J. 2016:47(2):564-74.

27. Borisov SE, Dheda K, Enwerem M, Romero Leyet R, D'Ambrosio L, Centis R, et al. Effectiveness and safety of bedaquiline-containing regimens in the treatment of MDR- and XDR-TB: a multicentre study. Eur Respir J. 2017;49(5): 1700387.

28. He G, Li Y, Chen X, Chen J, Zhang W. Prediction of treatment outcomes for multidrug-resistant tuberculosis by whole-genome sequencing. Int J Infect Dis. 2020;96:68-72.

\section{Publisher's Note}

Springer Nature remains neutral with regard to jurisdictional claims in published maps and institutional affiliations.
Ready to submit your research? Choose BMC and benefit from:

- fast, convenient online submission

- thorough peer review by experienced researchers in your field

- rapid publication on acceptance

- support for research data, including large and complex data types

- gold Open Access which fosters wider collaboration and increased citations

- maximum visibility for your research: over $100 \mathrm{M}$ website views per year

At $\mathrm{BMC}$, research is always in progress.

Learn more biomedcentral.com/submissions 